\title{
OPTIMAL CONTROL OF DIFFUSION EQUATION WITH FRACTIONAL TIME DERIVATIVE WITH NONLOCAL AND NONSINGULAR MITTAG-LEFFLER KERNEL
}

\author{
J.D. DJIDA, G.M. MOPHOU, AND I. AREA
}

\begin{abstract}
In this paper, we consider a diffusion equation with fractional-time derivative with nonsingular Mittag-Leffler kernel in Hilbert spaces. Existence and uniqueness of solution are proved by means of a spectral argument. The existence of solution is obtained for all values of the fractional parameter $\alpha \in(0,1)$. Moreover, by applying control theory to the fractional diffusion problem we obtain an optimality system which has also a unique solution.
\end{abstract}

\section{INTRODUCTION}

In some problems related with transport dynamics in complex systems, the anomalous diffusion appear in a natural way [1]. As a consequence, some classical differential equations have been studied in the context of fractional derivatives, such as diffusion equation, diffusion-advection or FokkerPlank type [1]. In [2] diffusion and wave equations together with appropriate initial condition(s) have been rewritten as integro-differential equations with time derivatives replaced by convolution with $t^{\alpha-1} / \Gamma(\alpha), \alpha=1,2$, respectively. These equations have been solved numerically by using different approaches (see e.g. [3, 4, 5] and references therein). In [17] the classical control theory has been applied to a fractional diffusion equation in a bounded domain, where the fractional time derivative was considered in a Riemann-Liouville sense.

We are interested in the analysis of the initial boundary value problem for a fractional time diffusion problem. Let $\Omega$ be an open and bounded subset of $\mathbb{R}^{d}(d \geq 1)$, with sufficiently smooth boundary $\partial \Omega$. Given $\alpha \in(0,1)$, a well behave function $f \in L^{2}(Q)$, and an initial datum $y^{0} \in L^{2}(\Omega)$, we seek $y$ such that

$$
\begin{cases}{ }_{0}^{\mathrm{abc}} D_{t}^{\alpha} y(x, t)-(\mathcal{A} y)(x, t)=f, & \text { for }(x, t) \in Q=\Omega \times(0, T), \\ y(x, 0)=y^{0}(x), & \text { for } x \in \Omega, \\ y(x, t)=0, & \text { on } \partial \Omega \times(0, T),\end{cases}
$$

where ${ }_{a}^{\text {abc }} D_{t}^{\alpha}$ for $\alpha \in(0,1)$ denotes the Atangana-Baleanu fractional derivative of order $\alpha$ in the sense of Caputo [6] with respect to the time $t$, which is formally defined in section 2 . In the cases of single and multiple fractional time Caputo derivatives, similar problems to (1.1) have been already studied. For the Caputo fractional derivative there exists a large and rapidly growing number of publications (see e.g. [14, 15, 16, 17, 18, 19] and references therein). Moreover, in [17] the Caputo fractional derivative was used to obtain an optimality system for the optimal control problem.

We would like to emphasize here that the existence and uniqueness of solutions for the fractional diffusion equation of type (1.1) when the Caputo fractional-time derivative is considered, have been obtained for $\alpha \in(1 / 2,1)$ [16, 17, 23]. In this work using the fractional-time derivative with the nonsingular Mittag-Laffler kernel, we have obtained the existence and uniqueness results for the fractional diffusion equation of type (1.1) for all the values $\alpha \in(0,1)$.

Furthermore, as for as the optimal control of the problem (1.1), one can refer to the methods of the Lagrange multiplier technique for the Caputo and Riemann fractional time derivative presented by

The first author grateful for the facilities provided by the German research Chairs and the Teacher Training Program of AIMS-Cameroon. The first author is also indebted to the AIMS-Cameroon 2017-2018 Tutor fellowship. The second author was supported by the Alexander von Humboldt foundation, under the programme financed by the BMBF entitled "German research Chairs". The work of the third author has been partially supported by the Agencia Estatal de Innovación (AEI) of Spain under grant MTM2016-75140-P, cofinanced by the European Community fund FEDER, and Xunta de Galicia, grants GRC 2015-004 and R 2016/022. 
different authors (see e.g. [16, 17, 23, 24, 25, 26] and references therein). We are interested in finding the control $\hat{u} \in L^{2}(Q)$ that minimizes the cost function

$$
\mathcal{J}(v)=\left\|y(v)-z_{d}\right\|_{L^{2}(\mathcal{Q})}^{2}+\frac{\mathcal{N}}{2}\|v\|_{L^{2}(Q)}^{2}, \quad z_{d} \in L^{2}(Q), \quad \mathcal{N}>0
$$

subject to the problem (1.1) where the the Atangana-Baleanu fractional-time derivative is involved. In other words we are interested in finding the control parameter $\hat{u} \in L^{2}(Q)$ such that the functional

$$
\mathcal{J}(\hat{u})=\inf _{v \in L^{2}(Q)} \mathcal{J}(v)
$$

holds true.

This manuscript is structured as follows: In section 2 we collect notation, definitions, and preliminary results regarding the solution representation and we compute the integration by parts involving the Atangana-Baleanu fractional-time derivative. Consequently the weak formulation of the (1.1) is obtained. Section 3, is dedicated to the sketch of proof of the existence and uniqueness of solution of the weak formulation problem. More precisely we prove Theorem 3.1 as well as some corollaries. Moreover, section 4 is devoted to an optimal control problem given by Theorem 4.2.

\section{Preliminaries}

In this section, we collect some notations on the functional spaces, useful properties on the MittagLeffler function and the fractional time derivatives, regularity results for the fractional diffusion (1.1), and basic estimates.

2.1. Notations. Let $L^{2}$ be the usual Hilbert space equipped with the scalar product $(\cdot, \cdot)$, and $H^{\ell}(\Omega)$, $H_{0}^{m}(\Omega)$ denote the usual Sobolev spaces. In order to make things clear, we define the second order elliptic operator as

$$
\mathcal{A} y:=-\Delta y, \text { for } x \in \bar{\Omega}, \quad \mathcal{D}(A)=H^{2}(\Omega) \cap H_{0}^{1}(\Omega) .
$$

Since $\mathcal{A}$ is an uniformly elliptic operator, the spectrum of $\mathcal{A}$ is composed of eigenvalues $\left\{\lambda_{n}\right\}_{n=1}^{\infty}$ and its corresponding orthogonal eigenfunctions $\left\{\varphi_{n}\right\}_{n=1}^{\infty}$ which belong to $H^{2}(\Omega) \cap H_{0}^{1}(\Omega)$, such that $\mathcal{A} \varphi_{n}=\lambda_{n} \varphi_{n}$.

2.2. Atangana-Baleanu fractional times derivatives: definitions and some properties. We first start by recalling the Mittag-Leffler function $E_{\alpha, \beta}(z)$ which will be used extensively throughout this work and is defined below

$$
E_{\alpha, \beta}(z)=\sum_{k=0}^{\infty} \frac{z^{k}}{\Gamma(k \alpha+\beta)}, \quad z \in \mathbb{C},
$$

where $\Gamma(\cdot)$ denotes the Gamma function defined as

$$
\Gamma(z)=\int_{0}^{\infty} t^{z-1} e^{-t} d t, \quad \Re(z)>0 .
$$

The Mittag-Leffler function is a two-parameter family of entire functions of $z$ of order $\alpha^{-1}[7$, pp. 42]. The exponential function is a particular case of the Mittag-Leffler function, namely $E_{1,1}(z)=e^{z}$, $[7,9]$. As presented in [10], two important members of this family are $E_{\alpha, 1}\left(-\lambda t^{\alpha}\right)$ and $t^{\alpha-1} E_{\alpha, \alpha}\left(-\lambda t^{\alpha}\right)$, which occur in the solution operators for the initial value problem (1.1).

Next we also recall the generalized Mittag-Leffler function [7, 9] defined as

$$
\mathcal{E}_{\alpha, \beta}^{\rho}(z)=\sum_{k=0}^{\infty} \frac{(\rho)_{k} z^{k}}{\Gamma(\alpha k+\beta) k !}, \quad \forall t \in \mathbb{C},
$$

where $(\rho)_{k}$ is the Pochhammer symbol defined as $(\rho)_{k}=\Gamma(\rho+k) / \Gamma(\rho)=\rho(\rho+1) \cdots(\rho+k-1)$.

Remark. Once could notice that for $\rho=1$ we have that

$$
\mathcal{E}_{\alpha, \beta}^{1}(z)=E_{\alpha, \beta}(z)
$$

Furthermore we recall the following Lemma from [7]. 
Lemma 2.1. Let $\alpha, \beta, \rho \in \mathbb{C}$ such that $\Re(\alpha)>0$ and $\Re(\beta)>0$. Then we have that

$$
\left(\frac{d}{d z}\right)^{k} \mathcal{E}_{\alpha, \beta}^{\rho}(z)=(\rho)_{k} \mathcal{E}_{\alpha, \alpha k+\beta}^{\rho+k}(z), \quad z \in \mathbb{C}, \quad k \in \mathbb{N}
$$

and in the other hand,

$$
\alpha \rho \mathcal{E}_{\alpha, \beta}^{\rho+1}(z)=(1+\alpha \rho-\beta) \mathcal{E}_{\alpha, \beta}^{\rho}(z)+\mathcal{E}_{\alpha, \beta-1}^{\rho}(z), \quad z \in \mathbb{C} .
$$

Let us recall some useful definitions of fractional derivatives in the sense of Atangana-Baleanu [6, 11].

Definition 2.2. For a given function $u \in H^{1}(a, T), T>a$, the Atangana-Baleanu fractional derivative of $u$ of order $\alpha$ in Caputo sense with base point $a$ is defined at a point $t \in(a, T)$ by

$$
{ }_{a}^{\mathrm{abc}} D_{t}^{\alpha} u(t)=\frac{B(\alpha)}{1-\alpha} \int_{a}^{t} u^{\prime}(\tau) E_{\alpha}\left[-\gamma(t-\tau)^{\alpha}\right] d \tau,
$$

where $\gamma=\alpha /(1-\alpha), E_{\alpha}(\cdot)$ stands for the Mittag-Leffler function, and $B(\alpha)=(1-\alpha)+\alpha / \Gamma(\alpha)$.

So from the definition on [12] we recall the following definition

Definition 2.3. For a given function $u \in H^{1}(a, T), T>t>a$, the Atangana-Baleanu fractional derivative of $u$ of order $\alpha$ in Caputo sense with base point $T$ is defined at a point $t \in(a, T)$ by

$$
\underset{T}{\mathrm{abc}} D_{t}^{\alpha} g(t)=-\frac{B(\alpha)}{1-\alpha} \int_{t}^{T} g^{\prime}(\tau) E_{\alpha}\left[-\gamma(\tau-t)^{\alpha}\right] d \tau
$$

Definition 2.4. Let $u \in H^{1}(a, T), T>a, \alpha \in(0,1)$. The Atangana-Baleanu fractional derivative of order $\alpha$ of $u$ in Riemann-Liouville sense with base point $a$ is defined at a point $t \in(a, T)$ as

$$
{ }_{a}^{\mathrm{abr}} D_{t}^{\alpha} u(t)=\frac{B(\alpha)}{1-\alpha} \frac{d}{d t} \int_{a}^{t} u(\tau) E_{\alpha}\left[-\gamma(t-\tau)^{\alpha}\right] d \tau .
$$

For $\alpha=1$ in (2.5), we consider the usual classical derivative $\partial_{t}$.

Furthermore the Atangana-Baleanu fractional integral of order $\alpha$ with base point $a$ is defined as

$$
I_{t}^{\alpha} u(t)=\frac{1-\alpha}{B(\alpha)} u(t)+\frac{\alpha}{B(\alpha) \Gamma(\alpha)} \int_{a}^{t} u(\tau)(t-\tau)^{\alpha-1} d \tau
$$

Notice that if $\alpha=0$ in (2.8) we recover the initial function, and if $\alpha=1 \mathrm{in}$ (2.8) we obtain the ordinary integral.

Next we recall the following lemma which has been stated and proved in [8, Theorem 1.6]

Lemma 2.5. Let $\alpha \in(0,1)$ and $\beta \in \mathbb{R}$ be arbitrary, and $\mu$ is such that $\frac{\alpha \pi}{2}<\mu<\min (\pi, \alpha \pi)$. Then there exists a real constant $C=C(\alpha, \beta, \mu)>0$ such that

$$
\left|E_{\alpha, \beta}(z)\right| \leq \frac{C}{1+|z|}, \quad \mu \leq|\arg (z)| \leq \pi .
$$

Lemma 2.6. Let set $\eta:[0, T] \rightarrow \mathbb{R}$, then for all $\alpha \in(0,1)$ we the equivalence relation hold true

$$
{ }_{T}^{a b c} D_{t}^{\alpha} \eta(T-t)={ }_{0}^{a b c} D_{t}^{\alpha} \eta(t) .
$$

Proof. The well-posedness of (2.10) follows from a change of variables. Let us set $\eta:[0, T] \rightarrow \mathbb{R}$, and let us define by $\widetilde{\eta}(t):=\eta(T-t)$. Notice that

$$
\widetilde{\eta}^{\prime}(t)=-\eta^{\prime}(T-t)
$$

Next by making change of variables $t \rightarrow T-t$ in (2.6), we get

$$
{ }_{0}^{\mathrm{abc}} D_{T-t}^{\alpha} \eta(T-t)=\underset{T}{\mathrm{abc}} D_{t}^{\alpha} \widetilde{\eta}(t)=-\frac{B(\alpha)}{1-\alpha} \int_{T-t}^{T} \eta^{\prime}(\tau) E_{\alpha}\left[-\gamma(\tau-(T-t))^{\alpha}\right] d \tau,
$$


setting $\omega=T-\tau$, we get that

$$
\begin{aligned}
& \underset{T}{\mathrm{abc}} D_{t}^{\alpha} \widetilde{\eta}(t)=\frac{B(\alpha)}{1-\alpha} \int_{T-t}^{T} \eta^{\prime}(T-\omega) E_{\alpha}\left[-\gamma(t-\omega)^{\alpha}\right] d \omega \\
& =\frac{B(\alpha)}{1-\alpha} \int_{0}^{t} \eta^{\prime}(T-\omega) E_{\alpha}\left[-\gamma(t-\omega)^{\alpha}\right] d \omega_{T}^{\mathrm{abc}} D_{t}^{\alpha} \widetilde{\eta}(t) \\
& =-\frac{B(\alpha)}{1-\alpha} \int_{0}^{t} E_{\alpha}\left[-\gamma(t-\omega)^{\alpha}\right] \widetilde{\eta}^{\prime}(\omega) d \omega,
\end{aligned}
$$

This means that

$$
{ }_{T}^{\mathrm{abc}} D_{t}^{\alpha} \widetilde{\eta}(t)=-{ }_{0}^{\mathrm{abc}} D_{t}^{\alpha} \widetilde{\eta}(t)
$$

As a consequence, the backwards in time with the fractional-time derivative with nonsingular MittagLeffler kernel at the based point $T$ is equivalently written as a forward in time operator with the fractional-time derivative with nonsingular Mittag-Leffler kernel $-{ }_{0}^{\mathrm{abc}} D_{t}^{\alpha}$.

2.3. Solution representation. Under the functional spaces introduced in subsection 2.1, we can associate with $u(x, t)$ a map $u(\cdot):(0, T) \rightarrow L^{2}(\Omega)$ by

$$
u(t)(x)=u(x, t), \quad 0<t<T, \quad x \in \Omega .
$$

Let us now multiply (1.1) by a function $\vartheta \in H_{0}^{1}(\Omega)$ and by integration by parts on $\Omega$, we get that

$$
\int_{\Omega}^{\mathrm{abc}}{ }_{0}^{\alpha} u(x, t) \vartheta(x) d x-\int_{\Omega} \Delta u(x, t) \vartheta(x) d x=\int_{\Omega} f(x, t) \vartheta(x) d x .
$$

By Green's formula, we have

$$
\int_{\Omega}{ }_{0}^{\mathrm{abc}} D_{t}^{\alpha} u(x, t) \vartheta(x) d x+\int_{\Omega} \nabla u(x, t) \nabla \vartheta(x) d x=\int_{\Omega} f(x, t) \vartheta(x) d x .
$$

Now we set for all

$$
\varphi, \psi \in L^{2}(\Omega), \quad(\varphi, \psi)_{L^{2}(\Omega)}=\int_{\Omega} \varphi(x) \psi(x) d x .
$$

The scalar product in $L^{2}(\Omega)$, with endowed norm $\|\varphi\|_{L^{2}(\Omega)}$. In the same spirit, we define the bilinear form in $H_{0}^{1}(\Omega)$ as

$$
a(\varphi, \psi)=\int_{\Omega} \nabla \varphi(x) \nabla \psi(x) d x, \quad \forall \varphi, \psi \in H_{0}^{1}(\Omega)
$$

so that we have $\|\varphi\|_{H_{0}^{1}(\Omega)}^{2}=a(\varphi, \psi)$.

Under the assumption that the operator $\mathcal{A}$ is an uniformly elliptic operator, the spectrum of $\mathcal{A}$ is composed of eigenvalues $\left\{\lambda_{k}\right\}_{k=1}^{\infty}$, and its corresponding orthogonal eigenfunctions $\left\{\lambda_{w_{k}}\right\}_{k=1}^{\infty}$ which belongs to $\mathcal{D}(A)$, such that

$$
a\left(w_{k}, p\right)=\lambda_{k}\left(w_{k}, r\right)_{L^{2}(\Omega)}, \quad \forall r \in H_{0}^{1}(\Omega) .
$$

Hence we have in this setting

$$
\|\varphi\|_{H_{0}^{1}(\Omega)}^{2}=\sum_{k=0}^{\infty} \lambda_{i}\left(\varphi, w_{i}\right)_{L^{2}(\Omega)}^{2}, \quad \forall \varphi \in H_{0}^{1}(\Omega) .
$$

As an immediate consequence, equation (2.13) can be written as follows

$$
\left({ }_{0}^{\mathrm{abc}} D_{t}^{\alpha} u(x, t), \vartheta\right)_{L^{2}(\Omega)}+a(u(t), \vartheta)_{L^{2}(\Omega)}=(f(t), \vartheta)_{L^{2}(\Omega)} .
$$

In this way, the problem (1.1) takes the representation as

$$
\begin{cases}\left({ }_{0}^{\mathrm{abc}} D_{t}^{\alpha} u(x, t), \vartheta\right)_{L^{2}(\Omega)}+a(u(t), \vartheta)_{L^{2}(\Omega)}=(f(t), \vartheta)_{L^{2}(\Omega)} & \text { in } \Omega, \forall \vartheta \in H_{0}^{1}(\Omega), \\ u(x, 0)=u^{0} & \text { for } x \in \Omega, \\ u(t)=0 & \text { on } \partial \Omega .\end{cases}
$$

To discuss the existence of uniqueness results of (1.1) or (2.14), we shall need some informations over the various time. We are interested in the solution of the fractional differential equation involving the Atangana-Baleanu fractional derivative, in the form of (2.14). To this end, we propose to solve 
the fractional integro-differential equation involving the Atangana-Baleanu fractional derivative in the form

$$
\left\{\begin{array}{l}
{ }_{0}^{\mathrm{abc}} D_{t}^{\alpha} y_{i}(t)+\lambda_{i} y_{i}(t)=f_{i}(t), \quad t \in(0, T) \\
y_{i}(0)=y_{i}^{0}
\end{array}\right.
$$

This type of fractional differential equation involving the fractional-time derivative with nonsingular Mittag-Leffler kernel has been solved in [22, Proposition 3.3] by the means of Laplace transform. By following the same procedure, we state the the next proposition

Proposition 2.7. Let $f \in L^{2}(\mathcal{Q})$, such that the Atangana-Baleanu fractional derivative exists. Then, the solution of differential equation (2.15), is given by

$$
y_{i}(t)=\zeta_{i} E_{\alpha}\left[-\gamma_{i} t^{\alpha}\right] y_{i}^{0}+\frac{(1-\alpha) \zeta_{i}}{B(\alpha)} f_{i}(t)+K_{i} \int_{0}^{t}(t-s)^{\alpha} E_{\alpha, \alpha}\left[-\gamma_{i}(t-s)^{\alpha}\right] f_{i}(s) d s,
$$

with

$$
\gamma_{i}=\frac{\alpha \lambda_{i}}{\left(B(\alpha)+(1-\alpha) \lambda_{i}\right)}, \quad \zeta_{i}=\frac{B(\alpha)}{\left(B(\alpha)+(1-\alpha) \lambda_{i}\right)}, \quad \text { and } \quad K_{i}=\left(\frac{\alpha \zeta_{i}}{B(\alpha) \Gamma(\alpha)}+\frac{(1-\alpha) \gamma_{i} \zeta_{i}}{B(\alpha)}\right) .
$$

2.4. Weak formulation of the problem. Next we state the following proposition which gives the weak formulation of the problem (1.1), that will be fundamental in our analysis.

Proposition 2.8. Let $\phi, y \in \mathcal{C}^{\infty}(\bar{Q})$. Then, we have

$$
\begin{aligned}
& \int_{\Omega} \int_{0}^{T}\left(\begin{array}{c}
a b c \\
0
\end{array} D_{t}^{\alpha} y(x, t)-\Delta y(x, t)\right) \phi(x, t) d x d t=\int_{0}^{T} \int_{\partial \Omega} y \frac{\partial \phi}{\partial \sigma} \phi d \sigma d t-\int_{0}^{T} \int_{\partial \Omega} \phi \frac{\partial y}{\partial \sigma} d \sigma d t \\
& +\int_{\Omega} \int_{0}^{T} y(x, t)\left(-{ }_{T}^{a b c} D_{t}^{\alpha} \phi(x, t)-\Delta \phi(x, t)\right) d t d x-\frac{B(\alpha)}{1-\alpha} \int_{\Omega} \int_{0}^{T} y(x, 0) E_{\alpha, \alpha}\left[-\gamma t^{\alpha}\right] \phi(x, t) d t d x \\
& +\frac{B(\alpha)}{1-\alpha} \int_{\Omega} \phi(x, T) \int_{0}^{T} y(x, t) E_{\alpha, \alpha}\left[-\gamma(T-t)^{\alpha}\right] d t d x
\end{aligned}
$$

Proof. For a given $\phi, y \in \mathcal{C}^{\infty}(\bar{Q})$, we have that

$$
\begin{aligned}
& \int_{\Omega} \int_{0}^{T}\left({ }_{0}^{\mathrm{abc}} D_{t}^{\alpha} y(x, t)-\Delta y(x, t)\right) \phi(x, t) d x d t \\
& =\int_{\Omega} \int_{0}^{T}{ }_{0}^{\mathrm{abc}} D_{t}^{\alpha} y(x, t) \phi(x, t) d x d t-\int_{\Omega} \int_{0}^{T} \Delta y(x, t) \phi(x, t) d x d t \\
& =(A)+(B)
\end{aligned}
$$

Let us compute each part.

\section{- computation of $(A)$ : (INTEgration by parts)}

Using the definition of the Atangana-Baleanu fractional derivative (2.5) at the based point $a=0$ we have the following

$$
\int_{0}^{T}{ }_{0}^{\mathrm{abc}} D_{t}^{\alpha} y(x, t) \phi(x, t) d t=\int_{0}^{T}\left[\frac{B(\alpha)}{1-\alpha} \int_{0}^{t} y^{\prime}(\tau) E_{\alpha}\left[-\gamma(t-\tau)^{\alpha}\right] d \tau\right] \phi(t) d t .
$$

We define by

$$
A_{1}:=\int_{0}^{t} y^{\prime}(\tau) E_{\alpha}\left[-\gamma(t-\tau)^{\alpha}\right] d \tau
$$

Then by computing $A_{1}$ by integration by parts, we get that

$$
A_{1}=y(t)-y(0) E_{\alpha}\left[-\alpha t^{\alpha}\right]-\int_{0}^{t} \gamma(t-\tau)^{\alpha-1} E_{\alpha, \alpha}\left[-\gamma(t-\tau)^{\alpha}\right] y(\tau) d \tau .
$$


Replacing $A_{1}$ by its value in (2.19) we get that

$$
\begin{aligned}
\int_{0}^{T}{ }_{0}^{\mathrm{abc}} D_{t}^{\alpha} y(x, t) \phi(x, t) d t & =\frac{B(\alpha)}{1-\alpha} \int_{0}^{T} y(x, t) \phi(x, t) d t-\frac{B(\alpha)}{1-\alpha} \int_{0}^{T} y(x, 0) E_{\alpha}\left[-\alpha t^{\alpha}\right] \phi(x, t) d t \\
& -\frac{B(\alpha)}{1-\alpha} \int_{0}^{T}\left[\int_{0}^{t} \gamma(t-\tau)^{\alpha-1} E_{\alpha, \alpha}\left[-\gamma(t-\tau)^{\alpha}\right] y(x, \tau) d \tau\right] \phi(x, t) d t \\
& :=A_{2}+A_{3}+A_{4},
\end{aligned}
$$

with

$$
\begin{aligned}
& A_{2}:=\frac{B(\alpha)}{1-\alpha} \int_{0}^{T} y(x, t) \phi(x, t) d t ; \quad A_{3}:=\frac{B(\alpha)}{1-\alpha} \int_{0}^{T} y(x, 0) E_{\alpha}\left[-\alpha t^{\alpha}\right] \phi(x, t) d t \\
& A_{4}:=\frac{B(\alpha)}{1-\alpha} \int_{0}^{T}\left[\int_{0}^{t} \gamma(t-\tau)^{\alpha-1} E_{\alpha, \alpha}\left[-\gamma(t-\tau)^{\alpha}\right] y(x, \tau) d \tau\right] \phi(x, t) d t .
\end{aligned}
$$

Let us compute $A_{4}$

$$
\begin{aligned}
A_{4} & :=-\frac{B(\alpha)}{1-\alpha} \int_{0}^{T}\left[\int_{0}^{t} \gamma(t-\tau)^{\alpha-1} E_{\alpha, \alpha}\left[-\gamma(t-\tau)^{\alpha}\right] y(x, \tau) d \tau\right] \phi(x, t) d t \\
& =\frac{B(\alpha)}{1-\alpha} \int_{0}^{T} y(x, \tau)\left[-\int_{\tau}^{T} \gamma(t-\tau)^{\alpha-1} E_{\alpha, \alpha}\left[-\gamma(t-\tau)^{\alpha}\right] \phi(x, t) d t\right] d \tau \\
& =\frac{B(\alpha)}{1-\alpha} \phi(x, T) \int_{0}^{T} y(x, t) E_{\alpha, \alpha}\left[-\gamma(T-t)^{\alpha}\right] d t-\frac{B(\alpha)}{1-\alpha} \int_{0}^{T} y(x, t) \phi(x, t) d t \\
& +\int_{0}^{T} y(x, t)\left[-\frac{B(\alpha)}{1-\alpha} \int_{t}^{T} E_{\alpha, \alpha}\left[-\gamma(t-\tau)^{\alpha}\right] \phi^{\prime}(x, \tau) d \tau\right] d t \\
& =\frac{B(\alpha)}{1-\alpha} \phi(x, T) \int_{0}^{T} y(x, t) E_{\alpha, \alpha}\left[-\gamma(T-t)^{\alpha}\right] d t-\frac{B(\alpha)}{1-\alpha} \int_{0}^{T} y(x, t) \phi(x, t) d t \\
& +\int_{0}^{T} y(x, t)_{T}^{\text {abc }} D_{t}^{\alpha} y(x, t) \phi(x, t) d t
\end{aligned}
$$

Hence replacing $A_{4}$ by its value in (2.20) we finally have that

$$
\begin{aligned}
& \int_{0}^{T} \underset{0}{\mathrm{abc}} D_{t}^{\alpha} y(x, t) \phi(x, t) d t=-\int_{0}^{T} y(x, t) \underset{T}{\mathrm{abc}} D_{t}^{\alpha} y(x, t) \phi(x, t) d t \\
& +\frac{B(\alpha)}{1-\alpha} \phi(x, T) \int_{0}^{T} y(x, t) E_{\alpha, \alpha}\left[-\gamma(T-t)^{\alpha}\right] d t-\frac{B(\alpha)}{1-\alpha} y(x, 0) \int_{0}^{T} E_{\alpha}\left[-\alpha t^{\alpha}\right] \phi(x, t) d t
\end{aligned}
$$

- COMputation of $(B)$

By a simple integration by parts we have that

$$
\begin{aligned}
(B) & :=-\int_{\Omega} \int_{0}^{T} \phi(x, t) \Delta u(x, t) d x d t=\int_{0}^{T} \int_{\partial \Omega} u \frac{\partial \phi}{\partial \sigma} \phi d \sigma d t-\int_{0}^{T} \int_{\partial \Omega} \phi \frac{\partial u}{\partial \sigma} d \sigma d t \\
& -\int_{\Omega} \int_{0}^{T} u(x, t) \Delta \phi(x, t) d t d x .
\end{aligned}
$$

Hence adding (2.21) to (2.22), we obtain the desired result (2.18).

Now using the conditions that $\phi(x, T)=0$, we have the following Corollary.

Corollary 2.9. Let $y$ be the solution of (1.1). Then, for any $\phi, y \in \mathcal{C}^{\infty}(\bar{Q})$ such that $\phi(x, T)=0$ in $\Omega$, we have that,

$$
\begin{aligned}
& \int_{\Omega} \int_{0}^{T}\left(\begin{array}{c}
a b c \\
0
\end{array} D_{t}^{\alpha} y(x, t)-\Delta y(x, t)\right) \phi(x, t) d x d t=\int_{0}^{T} \int_{\partial \Omega} y \frac{\partial \phi}{\partial \sigma} \phi d \sigma d t-\int_{0}^{T} \int_{\partial \Omega} \phi \frac{\partial y}{\partial \sigma} d \sigma d t \\
& +\int_{\Omega} \int_{0}^{T} y(x, t)\left(-\underset{T}{a b c} D_{t}^{\alpha} \phi(x, t)-\Delta \phi(x, t)\right) d t d x-\frac{B(\alpha)}{1-\alpha} \int_{\Omega} \int_{0}^{T} y(x, 0) E_{\alpha, \alpha}\left[-\gamma t^{\alpha}\right] \phi(x, t) d t d x .
\end{aligned}
$$




\section{Existence AND UNIQUENESS OF SOLUTION}

Let us consider the following problem, for $\alpha \in(0,1)$. Assume $\left.f \in L^{2}(Q)\right)$ and $y^{0} \in L^{2}(\Omega)$. We want to find $y \in L^{2}\left((0, T), H_{0}^{1}(\Omega)\right), y(x, 0) \in \mathcal{C}\left([0, T] ; H_{0}^{1}(\Omega)\right)$, such that $\forall \vartheta \in H_{0}^{1}(\Omega)$, we have

$$
\begin{cases}\left({ }_{0}^{\mathrm{abc}} D_{t}^{\alpha} y(t), \vartheta\right)_{L^{2}(\Omega)}+a(y(t), \vartheta)_{L^{2}(\Omega)}=(f(t), \vartheta)_{L^{2}(\Omega)} & \text { for all } t \in(0, T), \\ y(x, 0)=y^{0} & \text { for } x \in \Omega,\end{cases}
$$

Theorem 3.1. Let $\alpha \in(0,1)$. Assume $\left.f \in L^{2}(Q)\right), y^{0} \in L^{2}(\Omega)$ and the bilinear form a $(\cdot, \cdot)$ defined as in (2.12). Then the problem (1.1) or (3.1) has a unique solution $y \in\left(L^{2}\left((0, T) ; H_{0}^{1}(\Omega)\right)\right) \cap$ $\left(\mathcal{C}\left([0, T] ; L^{2}(\Omega)\right)\right)$ given by

$$
y(x, t)=\sum_{i=1}^{+\infty}\left\{\zeta_{i} E_{\alpha}\left[-\gamma_{i} t^{\alpha}\right] y_{i}^{0}+\frac{(1-\alpha) \zeta_{i}}{B(\alpha)} f_{i}(t)+K_{i} \int_{0}^{t}(t-s)^{\alpha-1} E_{\alpha, \alpha}\left[-\gamma_{i}(t-s)^{\alpha}\right] f_{i}(s) d s\right\} w_{i},
$$

where the constants $\gamma_{i}$ and $\zeta_{i}$ are defined as in (2.17). Moreover y satisfies the bounded conditions

$$
\|y\|_{L^{2}\left((0, T) ; H_{0}^{1}(\Omega)\right)} \leq \Lambda_{1}\left(\left\|y^{0}\right\|_{\left.H_{0}^{1}(\Omega)\right)}+\|f\|_{L^{2}(Q)}\right),
$$

where $\Lambda_{1}=\max \left(\mathcal{C}_{1}\left(\alpha, \lambda_{1}, T\right), \mathcal{C}_{2}\left(\alpha, \lambda_{1}, T\right)\right)$, with

$$
\mathcal{C}_{1}\left(\alpha, \lambda_{1}, T\right)=\frac{C B(\alpha)}{(1-\alpha)} \sqrt{\frac{6 T}{\lambda_{1}}}, \quad \mathcal{C}_{2}\left(\alpha, \lambda_{1}, T\right)=\sqrt{\frac{6 B^{2}(\alpha)}{(1-\alpha)^{2} \lambda_{1}}+\frac{12 C^{2} T^{2}}{\Gamma^{2}(\alpha)}\left(\frac{\Gamma^{2}(\alpha)+1}{\lambda_{1}}\right)},
$$

and

$$
\|y\|_{L^{2}(\Omega)} \leq \Lambda_{2}\left(\left\|y^{0}\right\|_{L^{2}(\Omega)}+\|f\|_{L^{2}(Q)}\right),
$$

where $\Lambda_{2}=\max \left(\mathcal{C}_{3}\left(\alpha, \lambda_{1}, T\right), \mathcal{C}_{4}\left(\alpha, \lambda_{1}, T\right)\right)$, with

$$
\mathcal{C}_{3}\left(\alpha, \lambda_{1}, T\right)=\frac{C B(\alpha)}{\lambda_{1}(1-\alpha)} \sqrt{6 T}, \quad \mathcal{C}_{4}\left(\alpha, \lambda_{1}, T\right)=\sqrt{\frac{6}{\lambda_{1}^{2}}+\frac{12 C^{2} T^{2}}{\Gamma^{2}(\alpha)}\left(\frac{\Gamma^{2}(\alpha)+1}{\lambda_{1}^{2}}\right)},
$$

providing that $y^{0} \in L^{2}(\Omega)$.

Proof. We first assume that the series defines in (2.13) converges uniformly. Let $\vartheta=w_{i}$ in (3.1) then we have that

$$
\left\{\begin{array}{l}
{ }_{0}^{\mathrm{abc}} D_{t}^{\alpha} y_{i}(t)+\lambda_{i} y_{i}(t)=f_{i}(t), \quad t \in(0, T) \\
y_{i}(0)=y_{i}^{0}
\end{array}\right.
$$

where we have used

$$
a\left(y(t), w_{i}\right)=\lambda_{i}\left(y(t), w_{i}\right)_{L^{2}(\Omega)}=\lambda_{i} y_{i} .
$$

We then recognize the system given by (3.5) which has been solved in subsection 2.3. So the solution of the fractional differential equation takes the form

$$
y_{i}(t)=\zeta_{i} E_{\alpha}\left[-\gamma_{i} t^{\alpha}\right] y_{i}^{0}+\frac{(1-\alpha) \zeta_{i}}{B(\alpha)} f_{i}(t)+K_{i} \int_{0}^{t}(t-s)^{\alpha-1} E_{\alpha, \alpha}\left[-\gamma_{i}(t-s)^{\alpha}\right] f_{i}(s) d s .
$$

Notice that the $\lambda_{i}$ are contained in $\zeta_{i}, \gamma_{i}$ and $K_{i}$ previously defined in subsection 2.3.

Now the next step is to look at the uniqueness of the solution. We will make it in two steps. We shall start by showing that the solution belongs to the space $L^{2}\left((0, T) ; H_{0}^{1}(\Omega)\right)$ and in the second step, we will show that the solution also belong to $\mathcal{C}\left([0, T] ; L^{2}(\Omega)\right)$, so that $y$ belongs to $L^{2}\left((0, T) ; H_{0}^{1}(\Omega)\right) \cap$ $\mathcal{C}\left([0, T] ; L^{2}(\Omega)\right)$.

Let defined by $\mathcal{V}_{m}$ a subspace of $H_{0}^{1}(\Omega)$ generated by $w_{1}, w_{2}, \ldots, w_{m}$. We want to find $y_{m}: t \in$ $(0, T] \rightarrow y_{m}(t) \in \mathcal{V}_{m}$ solution of fractional differential equation

$$
\begin{cases}\left({ }_{0}^{\mathrm{abc}} D_{t}^{\alpha}\left(y_{m}(t), \vartheta\right)\right)_{L^{2}(\Omega)}+a\left(y_{m}(t), \vartheta\right)_{L^{2}(\Omega)}=(f(t), \vartheta)_{L^{2}(\Omega)} & \text { for all } \vartheta \in \mathcal{V}_{m}, \\ y_{m}(x, 0)=y_{m}^{0} & \text { for } x \in \Omega .\end{cases}
$$


Since $y_{m}(t) \in \mathcal{V}_{m}$, we have

$$
y_{m}(t)=\sum_{i=1}^{m}\left(y(t), w_{i}\right)_{L^{2}(\Omega)} w_{i}=\sum_{i=1}^{m} y_{i}(t) w_{i}
$$

which could still be written in an explicit form as

$$
y_{m}(x, t)=\sum_{i=1}^{m}\left\{\zeta_{i} E_{\alpha}\left[-\gamma_{i} t^{\alpha}\right] y_{i}^{0}+\frac{(1-\alpha) \zeta_{i}}{B(\alpha)} f_{i}(t)+K_{i} \int_{0}^{t}(t-s)^{\alpha-1} E_{\alpha, \alpha}\left[-\gamma_{i}(t-s)^{\alpha}\right] f_{i}(s) d s\right\} w_{i} .
$$

The next step is to show $y_{m}(t)$ is a Cauchy sequence in the space $L^{2}\left((0, T) ; H_{0}^{1}(\Omega)\right)$. Let $m$ and $r$ be two natural numbers such that $r>m$. Then

$$
y_{r}(t)-y_{m}(t)=\sum_{i=m+1}^{r} y_{i}(t) w_{i} .
$$

But we also have that

$$
\begin{aligned}
& a\left(y_{r}(t)-y_{m}(t), y_{r}(t)-y_{m}(t)\right)=\sum_{i=m+1}^{r} \lambda_{i}\left(y_{i}(t)\right)^{2} \\
& =\sum_{i=m+1}^{r} \lambda_{i}\left\{\zeta_{i} E_{\alpha}\left[-\gamma_{i} t^{\alpha}\right] y_{i}^{0}+\frac{(1-\alpha) \zeta_{i}}{B(\alpha)} f_{i}(t)+K_{i} \int_{0}^{t}(t-s)^{\alpha-1} E_{\alpha, \alpha}\left[-\gamma_{i}(t-s)^{\alpha}\right] f_{i}(s) d s\right\}^{2} \\
& \leq 3 \sum_{i=m+1}^{r} \lambda_{i}\left[\zeta_{i} E_{\alpha}\left[-\gamma_{i} t^{\alpha}\right] y_{i}^{0}\right]^{2}+3 \sum_{i=m+1}^{r} \lambda_{i}\left[\frac{(1-\alpha) \zeta_{i}}{B(\alpha)} f_{i}(t)\right]^{2} \\
& +3 \sum_{i=m+1}^{r} \lambda_{i} K_{i}^{2}\left[\int_{0}^{t}(t-s)^{\alpha-1} E_{\alpha, \alpha}\left[-\gamma_{i}(t-s)^{\alpha}\right] f_{i}(s) d s\right]^{2} .
\end{aligned}
$$

Hence

$$
\int_{0}^{T}\left\|y_{r}(t)-y_{m}(t)\right\|_{L^{2}\left((0, T) ; H_{0}^{1}(\Omega)\right)}^{2} d t=\int_{0}^{T} a\left(y_{r}(t)-y_{m}(t), y_{r}(t)-y_{m}(t)\right) d t \leq 3\left(Z_{1}+Z_{2}+Z_{3}\right),
$$

where $Z_{1}, Z_{2}$ and $Z_{3}$ are defined respectively as

$$
\begin{aligned}
& Z_{1}:=\sum_{i=m+1}^{r} \lambda_{i} \int_{0}^{T}\left[\zeta_{i} E_{\alpha}\left[-\gamma_{i} t^{\alpha}\right] y_{i}^{0}\right]^{2} d t, \quad Z_{2}:=\sum_{i=m+1}^{r} \lambda_{i} \int_{0}^{T}\left[\frac{(1-\alpha) \zeta_{i}}{B(\alpha)} f_{i}(t)\right]^{2} d t, \\
& Z_{3}:=\sum_{i=m+1}^{r} \lambda_{i} K_{i}^{2} \int_{0}^{T}\left[\int_{0}^{t}(t-s)^{\alpha-1} E_{\alpha, \alpha}\left[-\gamma_{i}(t-s)^{\alpha}\right] f_{i}(s) d s\right]^{2} d t .
\end{aligned}
$$

Now the goal is to estimate each term $Z_{1}, Z_{2}$ and $Z_{3}$.

- Estimate of $Z_{1}$. Using the estimate of the Mittag-Leffler function provided in Lemma 2.5 we have that

$$
\begin{aligned}
Z_{1} & =\sum_{i=1+m}^{r} \lambda_{i} \int_{0}^{T}\left[\zeta_{i} E_{\alpha}\left[-\gamma_{i} t^{\alpha}\right] u_{i}^{0}\right]^{2} d t \leq 2 C^{2} \sum_{i=m+1}^{r} \lambda_{i} \zeta_{i}^{2}\left|u_{i}^{0}\right|^{2} \int_{0}^{T}\left(\frac{1}{1+\gamma_{i} t^{\alpha}}\right)^{2} d t \\
& \leq 2 C^{2} T \sum_{i=m+1}^{r} \lambda_{i} \zeta_{i}^{2}\left|u_{i}^{0}\right|^{2}
\end{aligned}
$$

Now we find the estimate $\lambda_{i} \zeta_{i}^{2}$ as

$$
\lambda_{i} \zeta_{i}^{2}=\frac{\lambda_{i} B^{2}(\alpha)}{\left(B(\alpha)+(1-\alpha) \lambda_{i}\right)^{2}} \leq \frac{\lambda_{i} B^{2}(\alpha)}{(1-\alpha)^{2} \lambda_{i}^{2}} \leq \frac{B^{2}(\alpha)}{(1-\alpha)^{2} \lambda_{i}} \leq \frac{B^{2}(\alpha)}{(1-\alpha)^{2} \lambda_{1}},
$$

since $\lambda_{i}$ are increasing. We finally get the estimate of $Z_{1}$ as

$$
Z_{1} \leq \frac{2 C^{2} B^{2}(\alpha)}{\lambda_{1}(1-\alpha)^{2}} T \sum_{i=m+1}^{r}\left|u_{i}^{0}\right|^{2} .
$$

- Estimate of $Z_{2}$. 


$$
\begin{aligned}
Z_{2} & =2 \sum_{i=m+1}^{r} \lambda_{i} \int_{0}^{T}\left[\frac{(1-\alpha) \zeta_{i}}{B(\alpha)} f_{i}(t)\right]^{2}=\frac{2(1-\alpha)^{2}}{B(\alpha)^{2}} \sum_{i=m+1}^{r} \lambda_{i} \zeta_{i}^{2}\left(\int_{0}^{T}\left\|f_{i}(t)\right\|^{2} d t\right) \\
& \leq \frac{2 B^{2}(\alpha)}{(1-\alpha)^{2} \lambda_{1}} \sum_{i=m+1}^{r}\left(\int_{0}^{T}\left|f_{i}(t)\right|^{2} d t\right) .
\end{aligned}
$$

- Estimate of $Z_{3}$. By applying the Cauchy-Schwarz inequality and by setting $\delta=t-s$ we have that

$$
\begin{aligned}
Z_{3} & =2 \sum_{i=m+1}^{r} \lambda_{i} K_{i}^{2} \int_{0}^{T}\left[\int_{0}^{t}(t-s)^{\alpha-1} E_{\alpha, \alpha}\left[-\gamma_{i}(t-s)^{\alpha-1}\right] f_{i}(s) d s\right]^{2} d t \\
& \leq 2 \sum_{i=m+1}^{r} \lambda_{i} K_{i}^{2} \int_{0}^{T}\left(\int_{0}^{t}\left(\delta^{\alpha-1} E_{\alpha, \alpha}\left[-\gamma_{i} \delta^{\alpha-1}\right]\right)^{2} d \delta\right)\left(\int_{0}^{t}\left\|f_{i}(s)\right\|^{2} d s\right) d t
\end{aligned}
$$

Now from Lemma 2.1 we have that

$$
\begin{aligned}
& \frac{d}{d \delta} E_{\alpha, 1}\left[-\gamma_{i} \delta^{\alpha}\right]=\frac{d}{d \delta} \mathcal{E}_{\alpha, 1}^{1}\left[-\gamma_{i} \delta^{\alpha}\right]=\mathcal{E}_{\alpha, \alpha+1}^{2}\left[-\gamma_{i} \delta^{\alpha}\right] \\
& =-\gamma_{i} \delta^{\alpha-1} E_{\alpha, \alpha}\left[-\gamma_{i} \delta^{\alpha}\right]=\frac{1}{\alpha} E_{\alpha, \alpha}\left[-\gamma_{i} \delta^{\alpha}\right] d \delta .
\end{aligned}
$$

Furthermore the estimate of $\lambda_{i} K_{i}^{2}$ is giving by

$$
\begin{aligned}
\lambda_{i} K_{i}^{2} & =\lambda_{i}\left(\frac{\alpha \zeta_{i}}{B(\alpha) \Gamma(\alpha)}+\frac{(1-\alpha) \gamma_{i} \zeta_{i}}{B(\alpha)}\right)^{2} \leq 2\left(\frac{\alpha^{2}}{B^{2}(\alpha) \Gamma^{2}(\alpha)} \lambda_{i} \zeta_{i}^{2}+\frac{(1-\alpha)^{2}}{B^{2}(\alpha)} \gamma_{i}^{2} \lambda_{i} \zeta_{i}^{2}\right) \\
& \leq \frac{2 \alpha^{2}}{\lambda_{1}(1-\alpha)^{2}}\left(1+\frac{1}{\Gamma^{2}(\alpha)}\right)
\end{aligned}
$$

Then we get that

$$
\begin{aligned}
Z_{3} & \leq \frac{4 \alpha^{2}}{\lambda_{1}(1-\alpha)^{2}}\left(1+\frac{1}{\Gamma^{2}(\alpha)}\right) \sum_{i=m+1}^{r} \frac{1}{\gamma_{i}^{2}} \int_{0}^{T}\left(\int_{0}^{t}\left(E_{\alpha, \alpha}\left[-\gamma_{i} \delta^{\alpha-1}\right]\right)^{2} d \delta\right)\left(\int_{0}^{t}\left\|f_{i}(s)\right\|^{2} d s\right) d t \\
& \leq \frac{4 C^{2}}{\lambda_{1}}\left(1+\frac{1}{\Gamma^{2}(\alpha)}\right) T \sum_{i=m+1}^{r} \int_{0}^{T}\left(\int_{0}^{T}\left\|f_{i}(t)\right\|^{2} d t\right) d t \\
& \leq \frac{4 C^{2} T^{2}}{\Gamma^{2}(\alpha)}\left(\frac{\Gamma^{2}(\alpha)+1}{\lambda_{1}}\right) \sum_{i=m+1}^{r} \int_{0}^{T}\left\|f_{i}(t)\right\|^{2} d t
\end{aligned}
$$

Finally, adding together the estimates $Z_{1}, Z_{2}$, and $Z_{3}$ we get

$$
\begin{aligned}
& \int_{0}^{T}\left\|y_{r}(x, t)-y_{m}(x, t)\right\|_{L^{2}\left((0, T) ; H_{0}^{1}(\Omega)\right)}^{2} d t \leq \frac{6 C^{2} B^{2}(\alpha)}{\lambda_{1}(1-\alpha)^{2}} T \sum_{i=m+1}^{r}\left|y_{i}^{0}\right|^{2} \\
& +\frac{6 B^{2}(\alpha)}{(1-\alpha)^{2} \lambda_{1}} \sum_{i=m+1}^{r}\left(\int_{0}^{T}\left|f_{i}(t)\right|^{2} d t\right)+\frac{12 C^{2} T^{2}}{\Gamma^{2}(\alpha)}\left(\frac{\Gamma^{2}(\alpha)+1}{\lambda_{1}}\right) \sum_{i=m+1}^{r} \int_{0}^{T}\left\|f_{i}(t)\right\|^{2} d t
\end{aligned}
$$

Thus we obtain

$$
\begin{aligned}
\int_{0}^{T}\left\|y_{r}-y_{m}\right\|_{H_{0}^{1}(\Omega)} & \leq \mathcal{C}_{1}\left(\alpha, \lambda_{1}, T\right)\left(\sum_{i=m+1}^{r}\left|y_{i}^{0}\right|^{2}\right)^{1 / 2} \\
& +\mathcal{C}_{2}\left(\alpha, \lambda_{1}, T\right)\left(\sum_{i=m+1}^{r} \int_{0}^{T}\left|f_{i}(t)\right|^{2} d t\right)^{1 / 2}
\end{aligned}
$$

with

$$
\mathcal{C}_{1}\left(\alpha, \lambda_{1}, T\right)=\frac{C B(\alpha)}{(1-\alpha)} \sqrt{\frac{6 T}{\lambda_{1}}}, \quad \mathcal{C}_{2}\left(\alpha, \lambda_{1}, T\right)=\sqrt{\frac{6 B^{2}(\alpha)}{(1-\alpha)^{2} \lambda_{1}}+\frac{12 C^{2} T^{2}}{\Gamma^{2}(\alpha)}\left(\frac{\Gamma^{2}(\alpha)+1}{\lambda_{1}}\right)} .
$$

As in the previous case, the norm in $L^{2}(\Omega)$ is computed as 
$(3.13)$

$$
\begin{aligned}
& \int_{0}^{T}\left\|y_{r}-y_{m}\right\|_{L^{2}(\Omega)}^{2} d t=\int_{0}^{T} \sum_{i=m+1}^{r}\left(y_{i}(t)\right)^{2} d t \\
& =\int_{0}^{T} \sum_{i=m+1}^{r}\left\{\zeta_{i} E_{\alpha}\left[-\gamma_{i} t^{\alpha}\right] y_{i}^{0}+\frac{(1-\alpha) \zeta_{i}}{B(\alpha)} f_{i}(t)+K_{i} \int_{0}^{t}(t-s)^{\alpha-1} E_{\alpha, \alpha}\left[-\gamma_{i}(t-s)^{\alpha}\right] f_{i}(s) d s\right\}^{2} d t \\
& \leq 3 \int_{0}^{T} \sum_{i=m+1}^{r}\left[\zeta_{i} E_{\alpha}\left[-\gamma_{i} t^{\alpha}\right] y_{i}^{0}\right]^{2} d t+3 \int_{0}^{T} \sum_{i=m+1}^{r}\left[\frac{(1-\alpha) \zeta_{i}}{B(\alpha)} f_{i}(t)\right]^{2} d t \\
& +3 \int_{0}^{T} \sum_{i=m+1}^{r} K_{i}^{2}\left[\int_{0}^{t}(t-s)^{\alpha-1} E_{\alpha, \alpha}\left[-\gamma_{i}(t-s)^{\alpha}\right] f_{i}(s) d s\right]^{2} d t .
\end{aligned}
$$

From the previous estimations in the first step of the proof, we have that

$$
\begin{aligned}
\int_{0}^{T}\left\|y_{r}-y_{m}\right\|_{L^{2}(\Omega)}^{2} d t & =\frac{6 C^{2} B^{2}(\alpha)}{(1-\alpha)^{2} \lambda_{1}^{2}} T \sum_{i=m+1}^{r}\left\|y_{i}^{0}\right\|^{2}+\frac{6}{\lambda_{1}^{2}} \sum_{i=m+1}^{r} \int_{0}^{T}\left\|f_{i}(t)\right\|^{2} \\
& +\frac{12 C^{2} T^{2}}{\Gamma^{2}(\alpha)}\left(\frac{\Gamma^{2}(\alpha)+1}{\lambda_{1}^{2}}\right) \sum_{i=m+1}^{r} \int_{0}^{T}\left\|f_{i}(t)\right\|^{2} d t
\end{aligned}
$$

Thus we obtain

$$
\begin{aligned}
& \sup _{t \in[0, T]}\left\|y_{r}-y_{m}\right\|_{L^{2}(\Omega)} \\
& \leq \mathcal{C}_{3}\left(\alpha, \lambda_{1}, T\right)\left(\sum_{i=m+1}^{r}\left|y_{i}^{0}\right|^{2}\right)^{1 / 2}+\mathcal{C}_{4}\left(\alpha, \lambda_{1}, T\right)\left(\sum_{i=m+1}^{r} \int_{0}^{T}\left|f_{i}(t)\right|^{2} d t\right)^{1 / 2},
\end{aligned}
$$

with

$$
\mathcal{C}_{3}\left(\alpha, \lambda_{1}, T\right)=\frac{C B(\alpha)}{\lambda_{1}(1-\alpha)} \sqrt{6 T}, \quad \mathcal{C}_{4}\left(\alpha, \lambda_{1}, T\right)=\sqrt{\frac{6}{\lambda_{1}^{2}}+\frac{12 C^{2} T^{2}}{\Gamma^{2}(\alpha)}\left(\frac{\Gamma^{2}(\alpha)+1}{\lambda_{1}^{2}}\right)} .
$$

From the fact that $y^{0} \in H_{0}^{1}(\Omega)$ and $f \in L^{2}(\mathcal{Q})$, we have that

$$
\lim _{m, r \rightarrow+\infty}\left(\sum_{i=m+1}^{r}\left|y_{i}^{0}\right|^{2}\right)^{1 / 2}=0, \quad \lim _{m, r \rightarrow+\infty}\left(\sum_{i=m+1}^{r} \int_{0}^{T}\left\|f_{i}(t)\right\|^{2} d t\right)^{1 / 2}=0 .
$$

Hence we have that

$$
\lim _{m, r \rightarrow+\infty} \int_{0}^{T}\left\|y_{r}-y_{m}\right\|_{L^{2}\left((0, T) ; H_{0}^{1}(\Omega)\right)}^{2}=0
$$

and

$$
\lim _{m, r \rightarrow+\infty} \int_{0}^{T}\left\|y_{r}-y_{m}\right\|_{L^{2}(\Omega)}^{2}=0
$$

which means that the sequence $\left(y_{m}\right)$ is Cauchy sequences in $L^{2}\left((0, T) ; H_{0}^{1}(\Omega)\right)$ and $\mathcal{C}\left([0, T] ; L^{2}(\Omega)\right)$.

Therefore we have that

$$
\begin{aligned}
& y_{m} \rightarrow y \quad \text { in } \quad L^{2}\left((0, T) ; H_{0}^{1}(\Omega)\right), \\
& y_{m} \rightarrow y \quad \text { in } \quad \mathcal{C}\left([0, T] ; L^{2}(\Omega)\right) .
\end{aligned}
$$

Now proceeding as in [23], one can prove by interpretation that the solution $y$ is equivalent to the initial problem (1.1). Furthermore, setting $\Lambda_{1}=\max \left(\mathcal{C}_{1}\left(\alpha, \lambda_{1}, T\right), \mathcal{C}_{2}\left(\alpha, \lambda_{1}, T\right)\right)$ and $\Lambda_{2}=$ $\max \left(\mathcal{C}_{3}\left(\alpha, \lambda_{1}, T\right), \mathcal{C}_{4}\left(\alpha, \lambda_{1}, T\right)\right)$, an using the estimates obtained at the beginning of the proof, we have that

$$
\begin{aligned}
\|y\|_{L^{2}\left((0, T) ; H_{0}^{1}(\Omega)\right)} & =\left(\int_{0}^{T}\|y\|_{H_{0}^{1}(\Omega)} d t\right)^{1 / 2} \\
& \leq \mathcal{C}_{1}\left(\alpha, \lambda_{1}, T\right)\left\|y^{0}\right\|_{L^{2}(\Omega)}+\mathcal{C}_{2}\left(\alpha, \lambda_{1}, T\right)\|f\|_{L^{2}(Q)} \\
& \leq \Lambda_{1}\left(\left\|y^{0}\right\|_{H_{0}^{1}(\Omega)}+\|f\|_{L^{2}(\mathcal{Q})}\right)
\end{aligned}
$$


and

$$
\begin{aligned}
\sup _{t \in[0, T]}\|y\|_{L^{2}(\Omega)} & \leq \mathcal{C}_{3}\left(\alpha, \lambda_{1}, T\right)\|y\|_{L^{2}(\Omega)}+\mathcal{C}_{4}\left(\alpha, \lambda_{1}, T\right)\|f\|_{L^{2}(Q)} \\
& \leq \Lambda_{2}\left(\left\|y^{0}\right\|_{L^{2}(\Omega)}+\|f\|_{L^{2}(Q)}\right)
\end{aligned}
$$

Hence we get the desired result, which ends the proof.

Remark. We would like to draw the attention of the reader that in the case for the Caputo fractionaltime derivative, the existence and uniqueness of solutions for the fractional diffusion equation of type (1.1), were obtained under the conditions that $\alpha \in(1 / 2,1)[16,17,23]$. But here in our case using the Atangana-Baleanu fractional-time derivative, we got the existence and uniqueness of solutions for the fractional diffusion equation of type (1.1) for all $\alpha \in(0,1)$.

Next we state two corollaries which give the estimates of the solution $y(t)$ in $L^{2}\left((0, T), H^{2}(\Omega) \cap H_{0}^{1}(\Omega)\right)$ for the case $f=0$ and in the case where the initial data $y^{0}=0$ respectively in the problem (1.1) or (3.1).

- First Case: $f=0$.

For $f=0$ in the problem (1.1) or (3.1) we have the following Corollary.

Corollary 3.2. Let $\alpha \in(0,1)$. Assume $f=0, y^{0} \in L^{2}(\Omega)$ and the bilinear form $a(\cdot, \cdot)$ defined as in (2.12). Then the problem (1.1) or (3.1) has a unique solution $y \in L^{2}\left((0, T), H^{2}(\Omega) \cap H_{0}^{1}(\Omega)\right)$ given by

$$
y(x, t)=\sum_{i=1}^{+\infty}\left\{\zeta_{i} E_{\alpha}\left[-\gamma_{i} t^{\alpha}\right] y_{i}^{0}\right\} w_{i},
$$

where the constant $\zeta_{i}$ is defined as in (2.17). Moreover $y$ satisfies the bounded conditions

$$
\int_{0}^{T}\|y\|_{H^{2}(\Omega)}^{2} d t=\|y\|_{L^{2}\left((0, T), H^{2}(\Omega)\right)}^{2} \leq \Lambda_{3}\left\|y^{0}\right\|_{H_{0}^{2}(\Omega)},
$$

where $\Lambda_{3}=\frac{C B(\alpha)}{(1-\alpha)} \sqrt{T}$.

Proof. Following the same procedure as in the first step for the proof of the Theorem 3.1, but now in $H_{0}^{2}(\Omega)$, the solution of the problem (1.1) or (3.1) for $f=0$ is given by

$$
y(t)=\sum_{i=1}^{+\infty}\left\{\zeta_{i} E_{\alpha}\left[-\gamma_{i} t^{\alpha}\right] y_{i}^{0}\right\} w_{i},
$$

where the constant $\zeta_{i}$ is defined as in (2.17). Furthermore the estimate of $y$ can be obtained as follows

$$
\begin{aligned}
\int_{0}^{T}\|y\|_{H^{2}(\Omega)}^{2} d t & =\int_{0}^{T} \sum_{i=m+1}^{r} \lambda_{i}^{2}\left(y_{i}(t)\right)^{2} d t=\int_{0}^{T} \sum_{i=m+1}^{r} \lambda_{i}^{2}\left\{\zeta_{i} E_{\alpha}\left[-\gamma_{i} t^{\alpha}\right] y_{i}^{0}\right\}^{2} d t \\
& \leq \sum_{i=m+1}^{r} \lambda_{i}^{2} \zeta_{i}^{2} \int_{0}^{T}\left(E_{\alpha}^{2}\left[-\gamma_{i} t^{\alpha}\right]\right)^{2}\left\|y_{i}^{0}\right\|^{2} d t \\
& \leq \frac{C^{2} B^{2}(\alpha)}{(1-\alpha)^{2}} T \sum_{i=m+1}^{r}\left\|y_{i}^{0}\right\|^{2} \\
& \leq \mathcal{C}_{5}(\alpha, T) \sum_{i=m+1}^{r}\left\|y_{i}^{0}\right\|^{2}
\end{aligned}
$$

- SECOND CASE: $y_{i}^{0}=0$.

For $y_{i}^{0}=0$ in the problem (1.1) or (3.1) we have the following Corollary.

Corollary 3.3. Let $\alpha \in(0,1)$. Assume $y_{i}^{0}=0$, and $f \in L^{2}(Q)$. Then the problem (1.1) or (3.1) has a unique solution $y \in L^{2}\left((0, T), H^{2}(\Omega) \cap H_{0}^{1}(\Omega)\right)$ given by

$$
y(x, t)=\sum_{i=1}^{+\infty}\left\{\frac{(1-\alpha) \zeta_{i}}{B(\alpha)} f_{i}(x, t)+K_{i} \int_{0}^{t}(t-s)^{\alpha-1} E_{\alpha, \alpha}\left[-\gamma_{i}(t-s)^{\alpha}\right] f_{i}(s) d s\right\} w_{i},
$$


where the constants $\gamma_{i}, \zeta_{i}$ and $K_{i}$ are defined as in (2.17). Moreover $y$ satisfies the bounded conditions

$$
\|y\|_{L^{2}\left((0, T), H^{2}(\Omega)\right)} \leq T \Lambda_{4}\|f\|_{L^{2}(Q)}
$$

where $\Lambda_{4}=\sqrt{\left(2+4 C^{2} T^{2}\left[1+\frac{1}{\Gamma^{2}(\alpha)}\right]\right)}$,

Proof. Let $\alpha \in(0,1)$. Assume $y_{i}^{0}=0$, and $f \in L^{2}(Q)$. Then the problem (1.1) or (3.1) has a unique solution $\left.y \in H^{2}(\Omega)\right)$ given by

$$
y(x, t)=\sum_{i=1}^{+\infty}\left\{\frac{(1-\alpha) \zeta_{i}}{B(\alpha)} f_{i}(t)+K_{i} \int_{0}^{t}(t-s)^{\alpha-1} E_{\alpha, \alpha}\left[-\gamma_{i}(t-s)^{\alpha}\right] f_{i}(s) d s\right\} w_{i},
$$

where the constants $\gamma_{i}, \zeta_{i}$ and $K_{i}$ are defined as in (2.17). Furthermore we compute the estimate of $y$ in the space $H^{2}(\Omega)$ as

(3.18)

$$
\begin{aligned}
\int_{0}^{T}\|y\|_{H^{2}(\Omega)}^{2} d t & =\int_{0}^{T} \sum_{i=m+1}^{r} \lambda_{i}^{2}\left\{\frac{(1-\alpha) \zeta_{i}}{B(\alpha)} f_{i}(t)+K_{i} \int_{0}^{t}(t-s)^{\alpha-1} E_{\alpha, \alpha}\left[-\gamma_{i}(t-s)^{\alpha}\right] f_{i}(s) d s\right\}^{2} d t \\
& \leq 2 \sum_{i=m+1}^{r} \lambda_{i}^{2} \int_{0}^{T}\left[\frac{(1-\alpha) \zeta_{i}}{B(\alpha)} f_{i}(t)\right]^{2} \\
& +2 \sum_{i=m+1}^{r} \lambda_{i}^{2} K_{i}^{2} \int_{0}^{T}\left(\int_{0}^{t}\left((t-s)^{\alpha-1} E_{\alpha, \alpha}\left[-\gamma_{i}(t-s)^{\alpha-1}\right]\right)^{2} d s\right)\left(\int_{0}^{t}\left\|f_{i}(s)\right\|^{2} d s\right) d t \\
& \leq 2 \sum_{i=m+1}^{r} \int_{0}^{T}\left\|f_{i}(t)\right\|^{2}+4 C^{2} T\left(1+\frac{1}{\Gamma^{2}(\alpha)}\right) \sum_{i=m+1}^{r} \int_{0}^{T}\left\|f_{i}(t)\right\|^{2} d t \\
& \leq \mathcal{C}_{6} \sum_{i=m+1}^{r} \int_{0}^{T}\left\|f_{i}(t)\right\|^{2} d t
\end{aligned}
$$

Now, we consider the following fractional differential equation:

$$
\left\{\begin{array}{l}
-{ }_{0}^{\mathrm{abc}} D_{t}^{\alpha} \eta(t)-\mathcal{A} \eta(t)=g(t), \quad t \in(0, T) \\
\eta(T)=0
\end{array}\right.
$$

where $\alpha \in(0,1), g \in L^{2}(Q)$. We state the following Lemma.

Lemma 3.4. Let $\alpha \in(0,1), g \in L^{2}(Q)$. Then problem (3.19) has a unique solution $\eta \in L^{2}(Q)$ given by

$$
\eta_{i}(x, t)=\sum_{i=1}^{+\infty}\left\{\frac{(1-\alpha) \zeta_{i}}{B(\alpha)} g(t)+K_{i} \int_{0}^{t}(t-s)^{\alpha} E_{\alpha, \alpha}\left[-\gamma_{i}(t-s)^{\alpha}\right] g(s) d s\right\} w_{i} .
$$

with the same constants as defined in (2.17). Moreover

$$
\left\|\eta_{i}\right\|_{L^{2}\left((0, T) ; H_{0}^{1}(\Omega)\right)} \leq \sqrt{\mathcal{C}_{2}}\|g\|_{L^{2}(Q)} .
$$

Proof. Using Lemma 2.6, we found that the backwards in time with the Atangana-Baleanu fractionaltime derivative at the based point $T$ is equivalently written as a forward in time operator with the Atangana-Baleanu fractional-time derivative at a based point zero time a negative sign. More precisely,

$$
\underset{T}{\mathrm{abc}} D_{t}^{\alpha} \widetilde{\eta}(t)=-{ }_{0}^{\mathrm{abc}} D_{t}^{\alpha} \widetilde{\eta}(t) .
$$

Using this relation and by making the change of variable $t \rightarrow T-t$ in (3.19), we obtain the fractional differential equation

$$
\left\{\begin{array}{l}
{ }_{0}^{\mathrm{abc}} D_{t}^{\alpha} \widetilde{\eta}(t)-\mathcal{A} \widetilde{\eta}(t)=\widetilde{g}(t), \quad T-t \in[0, T] \\
\eta(0)=0
\end{array}\right.
$$


with $\widetilde{g}(t)=g(T-t)$. This fractional differential equation can still be written in the short form as

$$
\left\{\begin{array}{l}
{ }_{0}^{\mathrm{abc}} D_{t}^{\alpha} \eta(\tau)-\mathcal{A} \eta(\tau)=g(\tau), \quad \tau \in[0, T], \\
\eta(0)=0
\end{array}\right.
$$

Next to show that (3.20) holds, we just apply the Laplace transform on (3.22) and get the inverse following the steps as in [22, Proposition 3.3], and by taking into account the fact that $\eta(0)=0$. Hence one get

$$
\eta(t)=\frac{(1-\alpha) \zeta_{i}}{B(\alpha)} g(t)+K_{i} \int_{0}^{t}(t-s)^{\alpha} E_{\alpha, \alpha}\left[-\gamma_{i}(t-s)^{\alpha}\right] g(s) d s,
$$

where $\zeta_{i}, \gamma_{i}$ and $\mathcal{C}_{2}$ were defined respectively in (2.17) and in (3.3)

Finally proceeding as for the proof of (3.2) in Theorem 3.1 once can obtain

$$
\left\|\eta_{i}\right\|_{L^{2}\left((0, T) ; H_{0}^{1}(\Omega)\right)} \leq \sqrt{\mathcal{C}_{2}}\|g\|_{L^{2}(Q)} \cdot
$$

\section{Optimal control PRoblem}

In this section, we want to approach the state $y(v)$ of the problem (1.1) by a desired state $z_{d}$ in controlling $v$.

Let $v \in L^{2}(Q)$. As it has been shown in the existence and uniqueness part, the problem (1.1) have a unique solution $y=y(v)$ which belongs to $L^{2}(Q)$. In this way, we can define can define the functional as

$$
\mathcal{J}(v)=\left\|y(v)-z_{d}\right\|_{L^{2}(Q)}^{2}+\frac{\mathcal{N}}{2}\|v\|_{L^{2}(Q)}^{2},
$$

where $z_{d} \in L^{2}(Q)$ and $\mathcal{N}>0$. We are interested on finding the control parameter, say, $\hat{u} \in L^{2}(Q)$ such that the functional

$$
\mathcal{J}(\hat{u})=\inf _{v \in L^{2}(Q)} \mathcal{J}(v)
$$

Now we state the following Proposition.

Proposition 4.1. Let consider the problem given by (1.1). Then there exists a unique optimal control u such that (4.2) holds true.

Proof. The functional $\mathcal{J}$ is continuous, coercive and convex. Therefore, there exist a control called $\hat{u}$. By calling $\hat{y}=\hat{y}(\hat{u})$, the associated state of $\hat{u}$ then its come that, $\hat{y}$ satisfies

$$
\begin{cases}\mathrm{abc} D_{t}^{\alpha} \hat{y}-\Delta \hat{y}=\hat{u} & \text { for }(x, t) \in Q, \\ \hat{y}(x, 0)=\hat{y}^{0}(x) & \text { for } x \in \Omega, \\ \hat{y}(x, t)=0 & \text { on } \partial \Omega \times(0, T),\end{cases}
$$

where $y \in L^{2}\left((0, T) ; H_{0}^{1}(\Omega)\right) \cap \mathcal{C}\left([0, T] ; L^{2}(\Omega)\right)$.

Remark. Another way of proving this result is to use minimizing sequences. The reader interested could follows the idea proposed by the second author in [17].

In the following we are concern with the interpretation of the Euler-Lagrange first order optimality condition with an adjoint problem defined by means of the backward Atangana-Baleanu fractionaltime derivative. As a result, we obtained an optimality system for the optimal control given by the following Theorem.

Theorem 4.2. If $\hat{u}$ is a solution of (4.2), then there exist $\eta$ such that

$$
(\hat{u}, \hat{y}, \eta) \in L^{2}(Q) \times L^{2}\left((0, T) ; H_{0}^{1}(\Omega)\right) \times L^{2}\left((0, T) ; H^{2}(\Omega) \cap H_{0}^{1}(\Omega)\right),
$$

satisfies the following optimality system

$$
\left\{\begin{aligned}
-{ }_{0}^{a b c} D_{t}^{\alpha} \hat{y}-\Delta \hat{y}=\hat{u} & \text { in } Q, \\
\hat{y}(x, 0)=\hat{y}^{0} & \text { in } \Omega, \\
\hat{y}(x, t)=0 & \text { on } \partial \Omega \times(0, T) .
\end{aligned}\right.
$$




$$
\begin{aligned}
& \left\{\begin{aligned}
-{ }_{T}^{a b c} D_{t}^{\alpha} \eta-\Delta \eta & =\hat{y}-z_{d} & & \text { in } Q, \\
\eta(T) & =0 & & \text { in } \Omega \\
\eta & =0 & & \text { on } \partial \Omega \times(0, T) .
\end{aligned}\right. \\
& \hat{u}=-\frac{\eta}{N} \quad \text { in } \quad Q .
\end{aligned}
$$

Proof. In order to prove the theorem, we start by expressing the Euler-Lagrange optimality conditions which characterize the optimal control $\hat{u}$. We means

$$
\left.\frac{d}{d \mu} \mathcal{J}(\hat{u}+\mu(v-\hat{u}))\right|_{\mu=0}, \quad \text { for all } \phi \in L^{2}(Q) .
$$

The state $z(v-\hat{u}) \in L^{2}\left((0, T) ; H^{2}(\Omega) \cap H_{0}^{1}(\Omega)\right)$ associated to the control $(v-\hat{u}) \in L^{2}(Q)$ is a solution of

$$
\left\{\begin{aligned}
{ }_{0}^{\mathrm{abc}} D_{t}^{\alpha} z-\Delta z & =v-\hat{u} & & \text { in } Q, \\
z(0, v-\hat{u}) & =0 & & \text { in } \Omega, \\
z & =0 & & \text { on } \partial \Omega \times(0, T) .
\end{aligned}\right.
$$

Now we find the following behaviour of our functional when $\mu$ converges to 0 .

$$
\lim _{\mu \rightarrow 0} \frac{\mathcal{J}(\hat{u}+\mu(v-\hat{u}))-\mathcal{J}(\hat{u})}{\mu}=0, \quad \text { for all } v \in L^{2}(Q) .
$$

Using the functional defined in (4.1) we have that

$$
\begin{aligned}
\mathcal{J}(\hat{u}+\mu(v-\hat{u})) & =\frac{\mathcal{N}}{2}(\hat{u}+\mu(v-\hat{u})(\hat{u}+\mu(v-\hat{u}) \\
& +\frac{1}{2}\left(\hat{y}(\hat{u})-z_{d}+\mu \hat{y}(v-\hat{u})\right)\left(\hat{y}(\hat{u})-z_{d}+\mu \hat{y}(v-\hat{u})\right) \\
& =\frac{\mathcal{N}}{2}\|\hat{u}\|_{L^{2}(Q)}^{2}+\mu \mathcal{N} \hat{u}(v-\hat{u})+\mu^{2} \frac{\mathcal{N}}{2}\|v-\hat{u}\|_{L^{2}(Q)}^{2} \\
& \left.+\frac{1}{2} \| \hat{y}(\hat{u})-z_{d}\right) \|_{L^{2}(Q)}^{2}+\mu\left(\hat{y}(\hat{u})-z_{d}\right)(\hat{y}(v-\hat{u})) \\
& +\frac{\mu^{2}}{2}\|\hat{y}(v-\hat{u})\|_{L^{2}(Q)}^{2}
\end{aligned}
$$

Hence we get that

$$
\lim _{\mu \rightarrow 0} \frac{\mathcal{J}(\hat{u}+\mu(v-\hat{u}))-\mathcal{J}(\hat{u})}{\mu}=z\left(\hat{y}(\hat{u})-z_{d}\right)+\mathcal{N} \hat{u}(v-\hat{u})=0, \quad \text { for all } v \in L^{2}(Q) .
$$

Now integrating over $Q$ we get that

$$
\int_{0}^{T} \int_{\Omega} z\left(\hat{y}(\hat{u})-z_{d}\right) d x d t+\mathcal{N} \int_{0}^{T} \int_{\Omega} \hat{u}(v-\hat{u}) d x d t=0 \quad \text { for all } v \in L^{2}(Q) .
$$

In order to make the interpretation (4.10), we consider the adjoint state equation

$$
\left\{\begin{aligned}
-{ }_{T}^{\mathrm{abc}} D_{t}^{\alpha} \eta-\Delta \eta & =\hat{y}-z_{d} & & \text { in } Q, \\
\eta(T) & =0 & & \text { in } \Omega, \\
\eta & =0 & & \text { on } \partial \Omega \times(0, T) .
\end{aligned}\right.
$$

But since $\hat{y}-z_{d} \in L^{2}\left((0, T), H_{0}^{1}(\Omega)\right)$, applying the Lemma 3.4, we deduce that the problem given by (4.11) has a unique solution in $L^{2}\left((0, T) ; H^{2}(\Omega) \cap H_{0}^{1}(\Omega)\right)$. In this scenario, by multiplying the state equation system (4.8) by the solution $\eta$ of (4.11), we obtain by 2.9 that

$$
\begin{aligned}
\int_{0}^{T} \int_{\Omega}\left({ }_{0}^{\mathrm{abc}} D_{t}^{\alpha} z-\Delta z\right) \eta d x d t & =\int_{0}^{T} \int_{\Omega}\left(-\underset{T}{\mathrm{abc}} D_{t}^{\alpha} \eta-\Delta \eta\right) z d x d t \\
& =\int_{0}^{T} \int_{\Omega}\left(\hat{y}(u)-z_{d}\right) z d x d t .
\end{aligned}
$$

Hence one get the following relation from (4.8) and (4.10), we deduce that

$$
\int_{0}^{T} \int_{\Omega}(v-\hat{u}) \eta d x d t=-\mathcal{N} \int_{0}^{T} \int_{\Omega}(v-\hat{u}) \hat{u} d x d t \quad \text { for all } v \in L^{2}(Q) .
$$


We then get finally that

$$
\hat{u}=-\frac{\eta}{\mathcal{N}} \quad \text { in } Q
$$

\section{Conclusion And Remarks}

In this work the existence and uniqueness of solution in $L^{2}(Q)$ were proved by means of a spectral argument and the control problem has also been studied. We have shown in the first step that the existence of solution were obtained for all values of the fractional parameter $\alpha \in(0,1)$, contrary to the Caputo and Riemann fractional-time derivative where the existence and uniqueness results were obtained for $\alpha \in(1 / 2,1)$ with $y^{0} \equiv 0$. This reveals one particularity of the fractional-time derivative with the nonsingular Mittag-Leffler function. Moreover, we have also shown that one can approach the state $y(v)$ of (1.1) by a desired state $z_{d}$ by controlling $v$ and compute the control $\hat{u}$ using the algorithm given by the optimality system and following the method of Lagrange. Let us also mention that by proceeding as in this work, one can also study the same problem by considering the Atangana-Baleanu fractional-time derivative in the Riemann-Liouville sense.

\section{REFERENCES}

[1] R. Metzler And J. Klafter. The random walk's guide to anomalous diffusion: a fractional dynamics approach. Physics Reports 339(1), 1-77, 2000.

[2] W. R. Schneider And W. Wyss. Fractional diffusion and wave equations. Journal of Mathematical Physics 30(1), 134-144, 1989.

[3] S.-L. Lei AND H.-W. Sun. A circulant preconditioner for fractional diffusion equations. Journal of Computational Physics 242, 715-725, 2013.

[4] Z.-P. HaO, Z.-Z. Sun, AND W.-R. CAO. A fourth-order approximation of fractional derivatives with its applications. Journal of Computational Physics 281, 787-805, 2015.

[5] H. Wang, K. Wang, and T. Sircar. A direct $O\left(N \log ^{2} N\right)$ finite difference method for fractional diffusion equations. Journal of Computational Physics 229, 8095-8104, 2010.

[6] A. Atangana And D. Baleanu. New fractional derivatives with non-local and non-singular kernel: theory and application to heat transfer model. Thermal Science 20(2), 763-769, 2016.

[7] A.A. Kilbas, H.M. Srivastava, and J.J. Trujillo. Theory and Applications of Fractional Differential Equations. Elsevier, Amsterdam, 2006.

[8] I. Podlubny. Fractional Differential Equations. Academic Press, San Diego, 1999.

[9] S. Samko, A. A. Kilbas, And O. Marichev. Fractional Integrals and Derivatives. Taylor \& Francis, 1993.

[10] B. Jin, R. Lazarov, and Z. Zhou. Error Estimates for a Semi-discrete Finite Element Method for Fractional Order Parabolic Equations. SIAM Journal on Numerical Analysis 51(1), 445-466, 2013.

[11] J.D. Djida, A. Atangana, And I. Area. Numerical Computation of a Fractional Derivative with Non-Local and Non-Singular Kernel. Math. Model. Nat. Phenom. 12(3), 4-13, 2017.

[12] T. Abdeljawad and D. Baleanu. Integration by parts and its applications of a new nonlocal fractional derivative with Mittag-Leffler nonsingular kernel. J. Nonlinear Sci. Appl. 10, 1098-1107, 2017.

[13] A. A. Kilbas, M. Saigo, And K. Saxena. Generalized Mittag-Leffler function and generalized fractional calculus operators. Int. Tran. Spec. Funct., 15(1), 31-49, 2004.

[14] E. Bazhlekova. Fractional Evolution Equation in Banach Spaces. Ph.D. Thesis. Eindhoven University of Technology, 2001.

[15] K. Diethelm and Y. Luchko. Numerical solution of linear multi-term initial value problems of fractional order. J. Comput. Anal. Appl. 6, 243-263, 2004.

[16] K. SАкамото And M. Yамамото, Initial value/boundary value problems for fractional diffusion-wave equations and applications to some inverse problems. J. Math. Anal. Appl. 382, 426-447, 2011.

[17] G. Mophou. Optimal control of fractional diffusion equation. Computers and Mathematics with Applications. 61, 68-78, 2011.

[18] Y. Luchko. Maximum principle for the generalized time-fractional diffusion equation. J. Math. Anal. Appl. 351, 218-223, 2009.

[19] Y. Luchko. Some uniqueness and existence results for the initial-boundary-value problems for the generalized time-fractional diffusion equation. Comput. Math. Appl. 59, 1766-1772, 2010.

[20] A. PAZy. Semigroups of Linear Operators and Applications to Partial Differential Equations. Springer, Berlin, 1983.

[21] S. Beckers and M. Yamamoto. Regularity and Unique Existence of Solution to Linear Diffusion Equation with Multiple Time-Fractional Derivatives. In: Bredies K., Clason C., Kunisch K., von Winckel G. (eds) Control and Optimization with PDE Constraints. International Series of Numerical Mathematics, vol 164. Birkhüser, Basel, 2013.

[22] J.D. Djida, A. Atangana, And I. Area. Parabolic problem with fractional time derivative with nonlocal and nonsingular Mittag-Leffler kernel. arXiv:1701.01479 , 2017.

[23] D. Baleanu, C. Joseph, and G. Mophou. Low regret control for a fractional wave equation with incomplete data. Advances in Difference Equations 240, 2016:240, (2016). 
[24] O.P. Agrawal. A general formulation and solution scheme for fractional optimal control problems. Nonlinear Dynam. 38,pp. 323-337, (2004).

[25] O.P. Agrawal. Formulation of Euler-Lagrange equations for fractional variational problems, J. Math.Anal. 272, 368-379, 2002.

[26] G.S.F. Frederico And D.F.M. Torres. Fractional optimal control in the sense of Caputo and the fractional Noether's Theorem, Int. Math. Forum 3(10), 479-493, 2008.

(Djida) Departamento de Análise Matemática, Universidade de Santiago de Compostela, 15782 Santiago de Compostela, Spain and African Institute for Mathematical Sciences (Aims), P.O. Box 608, Limbe Crystal Gardens, South West Region, Cameroon

E-mail address, Djida: jeandaniel.djida@aims-cameroon.org

(Mophou) African Institute for Mathematical Sciences (Aims), P.O. Box 608, Limbe Crystal Gardens, South West Region, Cameroon and Laboratoire C.E.R.E.G.M.I.A., Département de Mathématiques et Informatique, Université des Antilles et de la Guyane, Campus Fouillole, 97159 Pointe-À-Pitre, (FWi), GuADELOUPE

E-mail address, Mophou: gisele.mophou@aims-cameroon.org

(Area) Departamento de Matemática Aplicada II, E.E. Aeronáutica e do Espazo, Universidade de Vigo, Campus As Lagoas s/n, 32004 Ourense, Spain.

E-mail address, Area: area@uvigo.es 